Revista Perspectivas Online: Biológicas \& Saúde Novembro/2020, v.10, n.35, p.53-62 ISSN: 2236-8868 (Online) DOI: $10.25242 / / 8868103520202073$

\title{
INFLUÊNCIA DA CLASSE SOCIOECONÔMICA, TAMANHO DA FAMÍLIA E ANTROPOMETRIA NA IDADE DA MENARCA
}

\author{
Edson dos Santos Farias ${ }^{1 *}$, Wellington Roberto Gomes de Carvalho ${ }^{2}$, Josivana Pontes dos \\ Santos $^{1}$ \& Ivanice Fernandes Barcelos Gemelli ${ }^{1}$
}

FARIAS, E.S.; CARVALHO, W.R.G.; SANTOS, J.P.; \& GEMELLI, I.F.B. Influência da classe socioeconômica, tamanho da família e a antropometria na idade da menarca. Perspectivas Online: Biológicas \& Saúde, v.10, n.35, p.53-62, 2020.

\section{RESUMO}

O objetivo do estudo foi verificar a idade da menarca (IM) e comparar à classe social, antropometria pré e pós-menarca. Amostra com 1026 meninas de 10 a 18 anos, divididas em dois grupos: prémenarca $29,2 \% \quad(n=299)$ e pós-menarca $70,8 \% \quad(n=727)$. A IM foi obtida pelo método status quo. As comparações de diferenças entre a classe econômica e tamanho da família com a IM foi utilizada a análise de variância (ANOVA one-way), entre os grupos pré-menarca e pósmenarca o teste " $t$ " de Student. A média total da IM na classe social foi de $11,91 \pm$ 0,41 e tamanho da família $11,90 \pm 0,21$. As meninas de famílias classe A alta $(\mathrm{p}=0,02)$ atingiram menarca em torno de 6,0 a 8,0 meses mais cedo do que os seus pares de famílias de classe média e baixa. A comparação entre o pré e pós-menarca apresentaram diferenças significativas nas variáveis antropométricas $\mathrm{p}<0,001$. Com base nos achados as meninas de famílias de classe elevada parecem menstruar mais cedo do que as meninas de classe social inferior. $\mathrm{Na}$ antropometria, as meninas do pós-menarca foram significativamente mais altas e mais pesadas. Já, as meninas pré-menarca mostraram um corpo linear melhor refletido pelo índice ponderal.

Palavras-chave: Menarca; Adolescente; Antropometria; Escola; Classe social; Índice de massa corporal.

\footnotetext{
${ }^{1}$ Universidade Federal de Rondônia - UNIR - Centro de Estudo e Pesquisa em Saúde Coletiva - CEPESCO - BR 364, KM 9,5, Porto Velho, RO, CEP: 76801-059, Brasil;

${ }^{2}$ Universidade Federal de Uberlândia - UFU - Av. João Naves de Ávila, 2121 Bloco 1H, Santa Mônica, Urbelândia, MG, CEP: 38400902, Brasil.

(*) e-mail: edson.unir@unir.br
} 


\section{INFLUENCE OF SOCIOECONOMIC CLASS, FAMILY SIZE AND ANTHROPOMETRY IN THE AGE OF MENARCHE}

\section{Edson dos Santos Farias ${ }^{1 *}$, Wellington Roberto Gomes de Carvalho², Josivana Pontes dos Santos $^{1}$ \& Ivanice Fernandes Barcelos Gemelli ${ }^{1}$}

FARIAS, E.S.; CARVALHO, W.R.G.; SANTOS, J.P.; \& GEMELLI, I.F.B. Influence of socioeconomic class, family size and anthropometry in the age of menarche. Online Perspectives: Biological \& Health, v.10, n.35, p.53-62, 2020.

ABSTRACT

\begin{abstract}
The objective of the study was to verify the age of menarche (IM) and to compare social class, pre- and post-menarche anthropometry. Sample with 1026 girls aged 10 to 18 years, divided into two groups: pre-menarche $29.2 \%(\mathrm{n}=299)$ and post-menarche $70.8 \%(\mathrm{n}=727)$. IM was obtained using the status quo method. Comparisons of differences between economic class and family size with MI were used to analyze variance (one-way ANOVA), between the pre-menarche and post-menarche groups using the Student's " $\mathrm{t}$ " test. The total mean BM in the social class was $11.91 \pm 0.41$ and family size $11.90 \pm 0.21$. The Girls from upper class A
\end{abstract}

families from families with high class $(p=0.02)$ reached menarche around 6.0 to 8.0 months earlier than their peers from middle-class families and low. The comparison between the pre- and postmenarcheal adolescents showed significant differences in the anthropometric variables $\mathrm{p}<0.001$. Based on the findings, girls from high-class families seem to menstruate earlier than girls from lower social classes. In anthropometry, post-menarche girls were significantly taller and heavier. The pre-menarche girls, on the other hand, showed a better linear body reflected by the weight index.

Keywords: Adolescent; Anthropometry; School; Social class; Body mass index.

\footnotetext{
${ }^{1}$ Universidade Federal de Rondônia - UNIR - Centro de Estudo e Pesquisa em Saúde Coletiva - CEPESCO - BR 364, KM 9,5, Porto Velho, RO, CEP: 76801-059, Brasil;

${ }^{2}$ Universidade Federal de Uberlândia - UFU - Av. João Naves de Ávila, 2121 Bloco 1H, Santa Mônica, Urbelândia, MG, CEP: 38400902, Brasil

(*) e-mail: edson.unir@unir.br
} 


\section{INTRODUÇÃO}

Durante a adolescência, o corpo passa por profundas e rápidas transformações em decorrência das mudanças hormonais que aceleram o crescimento físico e também o desenvolvimento dos caracteres sexuais secundários (DENG et al., 2017).

Neste contexto, a idade da menarca não é apenas um reflexo do início da função ovariana, mas também, um preditor de frequência ovulatória (YERMACHENKOAND; DVORNYK; NONGENETIC, 2014). A idade em que um indivíduo atinge a menarca não depende unicamente do potencial genético, mas também do estado da saúde, que por sua vez é influenciado por fatores ambientais, como padrões gerais de vida e nutrição (SILVIA; BARROS FILH0, 2000).

Os achados de alguns estudos revelaram que a classe social familiar bem como o tamanho da família influencia na idade menarca (CARVALHO et al., 2007; ROMAN et al., 2009).

Estudos têm demonstrado associações significativas do índice de massa corporal (IMC) com a idade da menarca, e indicando uma precocidade na idade da menarca. Diversos fatores têm sido associados a este fato, sendo um deles o aumento da prevalência de obesidade, provocando nesses grupos uma menarca mais precoce influenciada pelo ganho de gordura (LAITINEN; POWER; JARVELIN, 2001).

O Recíproco do Índice Ponderal (RPI) é um indicador antropométrico e tem sido utilizado como uma ferramenta para estudar o físico da desnutrição e sobrepeso de crianças e adolescentes e medir a linearidade do corpo. O RIP é comumente utilizado em pediatria, porque os valores resultantes são consistentes em diferentes alturas, e possibilita estabelecer associação com a idade no início da menarca (TUNAU et al., 2012).

Diante disso, o objetivo deste estudo foi verificar a idade da menarca e comparar com a classe socioeconômica, tamanho da família e a antropometria.

\section{METODOLOGIA}

Estudo de delineamento transversal realizado na cidade de Porto Velho - RO, com 519.436 habitantes, localizada ao sudoeste da Amazônia Brasileira. A população total estimada de estudantes matriculados, e frequentando o ensino fundamental II e médio em Porto Velho foi de 26.546 estudantes do sexo feminino distribuídos na faixa etária de 10 a 18 anos de idade. Os dados foram levantados junto à Secretaria Estadual de Educação do estado de Rondônia (SEDUC) entre os meses de março a julho/2017.

Para o cálculo do tamanho da amostra considerou-se a prevalência para o desfecho de $50 \%$ para a pós-menarca (presença de menarca), intervalo de confiança de $95 \%$ (IC95\%), erro máximo aceitável de 3\%, efeito de delineamento (deff) igual a dois e acréscimo de $20 \%$ para compensar perdas e recusas. Com base nesses parâmetros, o tamanho da amostra foi estimado em 1.026 meninas adolescentes divididos em dois grupos: G1 = pré-menarca 29,2\% $(\mathrm{n}=299)$ e $\mathrm{G} 2$ = pós-menarca 70,8\% $(\mathrm{n}=727)$. A amostra foi selecionada por conglomerados em dois estágios: seleção sistemática de 25 escolas distribuídas proporcionalmente por tipo (públicas e privadas), tamanho (número de alunos) e região geográfica do município (norte, 
sul, leste, oeste); seleção aleatória de turmas para compor a amostra $(\mathrm{n}=125)$, distribuídas proporcionalmente por turno (matutino e vespertino) e série de ensino (ensino fundamental $\mathrm{II}=6^{\circ}$ ao $9^{\circ}$ e do ensino médio $=1^{\circ}, 2^{\circ}$ e $3^{\circ}$ anos $)$.

No presente estudo foram incluídas somente as adolescentes (10 a 18 anos) matriculadas na rede pública estadual de ensino e que se encontravam na sala de aula no dia da coleta de dados para preenchimento do questionário estruturado e realização das medidas antropométricas. Foram excluídas as meninas impossibilitadas de fazer as medidas antropométricas e aquelas com menos de 10 anos e com mais de 18 anos de idade.

O questionário utilizado para classificação da classe econômica foi o Critério de Classificação Econômica Brasil da Associação Nacional de Empresas de Pesquisa (ABEP, 2015), levando em conta os números de utensílios domésticos na residência e o grau de instrução dos pais, que após a contagem dos pontos foram classificadas em classes sociais A (classe alta), B (classe média), C, D e E (classe baixa). O tamanho da família foi classificado em pequena ( $\leq 2$ membros), média ( $>2 \mathrm{e} \leq 5$ membros) e grande ( $>5$ membros).

O procedimento para aplicação do questionário foi em sala de aula com a presença dos pesquisadores e do professor que o auxiliou. O questionário foi lido e explicado pelo entrevistador, e, no decorrer do seu preenchimento, as dúvidas foram sendo sanadas. Após o preenchimento, os questionários foram recolhidos para análise.

A idade da menarca foi obtida pelo método status quo que questionava a presença ou não de menstruação. Para as respostas positivas utilizou-se um questionário retrospectivo procurando identificar o dia, o mês e o ano de sua ocorrência. A maturação sexual foi obtida por autoavaliação com a comparação das fotos dos cinco estágios de desenvolvimento das mamas (M1 a M5), de acordo com os critérios descritos por Marshall e Tanner (1969). As meninas foram divididas em dois grupos pré-menarca considerada sem menstruação e pósmenarca incluídas as meninas com estágio M2 para cima com menstruação. O Recíproco do Índice Ponderal (RIP) foi calculado a partir da razão entre a estatura em centímetros $(\mathrm{cm})$ e a raiz cúbica da massa corporal em quilograma $(\mathrm{kg})[(\mathrm{RIP}=$ estatura $(\mathrm{cm}) / \mathrm{massa}$ corporal $\left.\left.(\mathrm{kg})^{1 / 3}\right)\right]$.

A massa corporal $(\mathrm{kg})$ e o índice de massa corporal (IMC) foram obtidos utilizando o equipamento de bioimpedância tetra-polar da marca $\operatorname{In-Body}{ }^{\circledR}$. Para a medida da estatura foi utilizado um estadiômetro portátil. A adolescente foi orientada a não fazer refeição por duas horas antes do exame, não praticar atividade física antes do exame, não estar em período menstrual e a permanecer apenas com a roupa de educação física, retirando calçados, meias e objetos metálicos. A técnica consistiu em ficar em pé sobre a plataforma, com o mínimo de roupa, posicionando os pés paralelos entre os quais flui uma corrente elétrica mínima que mede a resistência do corpo à passagem da corrente. Utilizado o protocolo recomendado pela National Institutes of Health (1996). A equipe composta pela pesquisadora médica e estudantes do curso de medicina, foi previamente treinada para aplicação do questionário bem como para executar as medidas. Toda a pesquisa foi realizada no próprio ambiente escolar.

Para o arquivo de dados e a análise estatística, foi utilizado o software IBM SPSS (Statistical Package for the Social Sciences) versão 20.0. O teste de normalidade ShapiroWilk foi utilizado para verificar a distribuição de curva de normalidade dos dados para as variáveis do estudo e para os grupos pré-menarca e pós-menarca. Para evidenciar as possíveis comparações de diferenças entre a classe econômica e tamanho da família com a idade da menarca, utilizou-se a análise de variância (ANOVA one-way) com post-hoc de Bonferroni. $\mathrm{Na}$ comparação de médias entre os grupos pré e pós-menarca com as variáveis antropométricas foi utilizado o teste " $\mathrm{t}$ " de Student para amostras independentes. A distribuição se manteve normal ( $p>0,05$ ). O nível de significância adotado foi de $p<0,05$.

O estudo foi aprovado pelo Comitê de Ética em Pesquisa (CEP) da Universidade Federal de Rondônia com o n. de parecer 729.901 e CAAE: 335422014.0.0000.5300.

Persp. online: biol. \& saúde, Campos dos Goytacazes, 35 (10) 53-62, 2020

https://ojs3.perspectivasonline.com.br 


\section{RESULTADOS}

Para a classe social o resultado da média total da IM foi de $11,91 \pm 0,41$ e para o tamanho da família na média total foi de $11,90 \pm 0,21$. Meninas de famílias de classe alta (A) atingiram a menarca seis a oito meses mais cedo do que os seus pares de famílias de classe média e baixa $(\mathrm{p}<0,05)$, respectivamente. Meninas de famílias pequenas tiveram a idade da menarca significativamente menor que as famílias classificadas de tamanho médio e grande $(\mathrm{p}<0,05)$ (Tabela 1).

Tabela 1: Comparação da idade da menarca $(\mathrm{G} 2)$ com a classe social e tamanha da família. Porto Velho (RO), Brasil, 2017

\begin{tabular}{cccc}
\hline Classe social & n (\%) & IM & Post Hoc (f) \\
\hline Alta & $199(16,4)$ & $11,44 \pm 1,27$ & a vs b=<0,02 \\
Média & $227(31,2)$ & $12,08 \pm 1,30$ & a vs c $=<0,05$ \\
Baixa & $301(41,4)$ & $12,22 \pm 1,27$ & b vs c $=<0,001$ \\
Total & 727 & $11,91 \pm 0,41$ & \\
\hline
\end{tabular}

Tamanho da

família

$\begin{array}{cccc}\text { Pequena } & 151(20,8) & 11,75 \pm 1,30 & \mathrm{~d} \text { vs e }=<0,01 \\ \text { Média } & 305(41,9) & 11,82 \pm 1,29 & \text { d vs } \mathrm{f}=<0,05 \\ \text { Grande } & 271(37,3) & 12,15 \pm 1,33 & \text { e vs } \mathrm{f}=<0,05 \\ \text { Total } & 727 & 11,90 \pm 0,21 & \end{array}$

Grupo pós-menarca $=\mathrm{G} 2$, Análise de Variância One-Way - ANOVA com Post-Hoc de Bonferroni: comparações múltiplas classe econômica = alta (a), média (b) e baixa (c) = a vs b, a vs c e b vs c, tamanho da família pequena (d), média (e) e grande (d) = $\mathrm{d}$ vs e, $\mathrm{d}$ vs $\mathrm{f}$ e e vs $\mathrm{f}$, valores significativos $\mathrm{p}<0,05$; valores expressos em $\mathrm{M}=$ média e DP=desvio padrão. Fonte: ABEP (http://www.abep.org/criterio-brasil) Classe A (A1 e A2) Alta, Classe B (B1, B2 e C) média e Classe D e E (baixa)

Na comparação dos dados antropométricos de meninas dos grupos pré (G1) e pósmenarca (G2) observou-se diferenças significativas para todas as variáveis antropométricas. com valores maiores para o grupo G2, com exceção para a variável RIP que o G1 foi maior (Tabela 2).

Tabela 2: Comparação de média, desvio padrão (M e DP) e intervalo de confiança $\left(\mathrm{IC}_{95 \%}\right)$ da massa corporal (MG), estatura (m), Índice de Massa Corporal (zIMC) e Recíproco do Índice Ponderal (RIP) dos grupos pré-menarca (G1)e pós-menarca (G2) das meninas escolares. Porto Velho (RO), Brasil, 2017

\begin{tabular}{|c|c|c|c|}
\hline \multirow[t]{2}{*}{ Variáveis } & $\begin{array}{c}\text { G1= Pré-menarca } \\
(n=299)\end{array}$ & $\begin{array}{c}\mathrm{G2}=\begin{array}{c}\text { Pós-menarca } \\
(\mathrm{n}=727)\end{array}\end{array}$ & t-valor \\
\hline & $\mathrm{M}$ e DP & M e DP & \\
\hline Massa corporal (kg) & $40,1 \pm 6,0$ & $47,7 \pm 7,0$ & $<0,001$ \\
\hline Estatura (m) & $1,46 \pm 5,2$ & $1,55 \pm 9,7$ & $<0,001$ \\
\hline $\operatorname{zIMC}\left(\mathrm{kg} / \mathrm{m}^{2}\right)$ & $15,9 \pm 2,0$ & $18,7 \pm 1,5$ & $<0,001$ \\
\hline $\operatorname{RIP}\left(\mathrm{kg} / \mathrm{cm}^{3}\right)$ & $45,0 \pm 1,5$ & $44,5 \pm 1,3$ & $<0,01$ \\
\hline
\end{tabular}




\section{DISCUSSÃO}

A média de idade com a ocorrência da menarca do presente estudo foi de 11,52 $\pm 1,35$ e sem ocorrência $11,12 \pm 0,93$. Na classe social $11,91 \pm 0,41$ e tamanho da família $11,90 \pm$ 0,21 . Os dados deste estudo indicam que na pós-menarca as meninas são significativamente acima do peso, altas e possuem maior zIMC que as suas homólogas que ainda não alcançaram a menarca.

Estudo ERICA que avaliou a idade da Menarca com 73.624 esstudantes, 40.803 meninas, das quais 37.390 referiram ter apresentado menarca, sendo a média da idade de ocorrência de 11,71 anos, mostrou que a ocorrência da IM foram menores naquelas com maior zIMC (sobrepeso e obesidade). Está ocorrência de presença de excesso de peso (HR = 1,28, IC95\% 1,21-1,36, $\mathrm{p}<0,001)$ e estudar em escola privada ( $\mathrm{HR}=1,06$, IC95\% 1,02-1,10, $\mathrm{p}=0,003$ ) estão associados à menarca. As meninas com excesso de peso (zIMC elevado) menstrual mais cedo (11,6 anos) do que as sem excesso de peso (12,3 anos) (SILVIA \& NUCCI, 2015).

O IMC elevado tem mostrado uma relação com a ocorrência mais precoce da menarca (GEMELLI; FARIAS; SOUZA, 2016). Estudos têm demonstrado que meninas pós-menarca com IMC elevado apresentam maior gordura corporal e sugere-se que a interação existente entre esses fatores podem estar relacionados à leptina (uma proteína derivada de gordura) que exerce efeito positivo na secreção de hormônio liberador de gonadotropina $(\mathrm{GnRH})$ pelo hipotálamo, resultando em estimulação do eixo pituitária-ovariana (KAUR, MEHTA; KAUR, 2015). Alguns estudos, ainda, concluem que a idade na menarca está mais relacionada à distribuição da gordura corpora (BANIK, 2011; KAUR, MEHTA; KAUR, 2015; GEMELLI; FARIAS; SOUZA, 2016).

Os dados do presente estudo são provenientes de meninas oriundas da cidade de Porto Velho, região Norte do Brasil (Latitude $8^{\circ} 45^{\prime} 43^{\prime \prime}$ Sul, Longitude 6354'7” Oeste) cujo clima é tropical superúmido de transição entre clima semiúmido da Região Centro-Oeste e o equatorial predominante na Região Norte, com quase 2.000 horas de sol por ano de acordo com Instituto Brasileiro de Geografia e Estatística (IBGE, 2017). Considerando as características geográficas, a idade de ocorrência da menarca no presente estudo pode ser explicada por fatores ambientais relacionados ao clima e à luz solar, condições que merecem atenção no contexto da variação de início da puberdade.

Um padrão de tempo sazonal da idade da menarca foi relatado por Matchock et al. (2004), em um estudo retrospectivo envolvendo 3.000 americanas, no qual os autores observaram fatores sazonais, como duração da luz solar e temperatura. Os autores chamam atenção para a sazonalidade da menarca, pois fatores como estresse ou fotoperíodo podem ter implicações para a saúde da mulher, considerando alguns dos riscos associados à menarca precoce. Outros estudos demostraram variações na idade da menarca de acordo com a latitude geográfica (MAO et al.; WULANDARI et al., 2017), sugerindo que a duração da luz do sol influencia a secreção de melatonina e modifica a época da menarca.

Sabe-se que os efeitos dos ritmos claro-escuros podem ser mediados pelo principal hormônio produzido e secretado pela glândula pineal, a melatonina, conhecida como 5metoxi-N-acetil-triptamina, que circula em altas concentrações à noite com importante papel na regulação do relógio biológico (WULANDARI et al., 2017). Costuma-se dizer, diante disso, que a melatonina é a molécula chave que controla o ciclo circadiano tanto em humanos 
quanto em animais (MAO et al., 2011), cujo controle é baseado conforme as condições de iluminação (WULANDARI et al., 2017).

O controle do relógio biológico e a síntese de melatonina ocorre a partir da conversão do aminoácido triptofano em 5-hidroxitriptofano (5-HTP) pela enzima triptofano hidroxilase 1 (TPH1). O 5-HTP é descarboxilado pela 5-HTP descarboxilase em serotonina, a qual é acetilada em $\mathrm{N}$-acetilserotonina (NAS) na reação catalisada pela enzima arilalquilamina $\mathrm{N}$ acetiltransferase (AA-NAT). Então, a NAS é convertida em melatonina pela enzima hidroxiindol-Ometiltransferase (HIOMT) (MAGANHIN et al., 2014; GEMELLI; FARIAS; SOUZA, 2016). Esses níveis de melatonina atingem o ápice na fase pré-pubere, para, em seguida, diminuírem progressivamente até o final da puberdade. Desta forma, a glândula pineal permitiria a ativação de pulsos de GnRH e, consequentemente, do eixo reprodutor (MAGANHIN et al., 2014).

No presente estudo, as meninas pré-menarca exibiram um IP (Índice Ponderal) médio significativamente maior (uma medida de linearidade do físico) do que seus pares pósmenarca. $\mathrm{O}$ valor normativo utilizado na interpretação do IP baseou-se em valores obtidos da própria população. Um IP mais elevado indica uma construção linear do corpo, isto é, menos peso para a altura. Este achado corrobora com o estudo de Kaur et al. (2015) e sugere que o tamanho do corpo exerce alguma influência sobre o tempo de maturação sexual e que a linearidade do corpo está associada à maturação tardia. A implicação clínica é que um físico mais linear (identificado por um IP médio alto) está associado a ocorrência da menarca mais tardia.

Considerando a estatura, as meninas que atingiram a menarca mais tardia tendem a serem maiores na fase adulta quando comparadas àquelas que tiveram menarca precoce (YERMACHENKOAND; DVORNYK; NONGENETIC, 2014). Esta relação é explicada pela fusão anterior da placa de crescimento em meninas que atingiram a menarca mais cedo devido ao aumento na produção de estrogênios ováricos associados à menarca (DENG et al., 2017).

Deve-se notar que os estudos diferem em delineamento e os métodos de coleta bem como a análise de dados variam de um estudo para outro, indicando a necessidade de ter cautela ao comparar a idade da menarca em diferentes estudos, em especial quando alguns não considerarem a classe socioeconômica. Cita-se aqui o estudo anterior na cidade de Benn, em que quase $2 \%$ das meninas atingiram a menarca antes dos 12 anos de idade (menarca precoce) (AHMED et al., 2016). Ainda o resultado publicado pelos autores do presente estudo quando não considerada a classe socioeconômica foi encontrada a média para a idade da menarca de 11,52 anos, para a mesma região norte do Brasil (GEMELLI et al., 2016). Considerando esta precocidade e as repercussões na adolescência e na idade adulta destas meninas, pode-se sugerir como possíveis fatores de risco para depressão na adolescência, excesso de peso, resistência à insulina e câncer de mama na idade adulta (KAUR; MEHTA; KAUR, 2015).

$\mathrm{O}$ achado do presente estudo relacionado à classe socioeconômica foi a média de idade da menarca de 11,91 $\pm 0,41$ anos. Estudo nacional em seus resultados encontrou a média da idade da ocorrência da menarca para os tercis alto, médio e baixo de nível socioeconômico $12,1 \pm 1,1 ; 12,3 \pm 1,2$ e 12,4 $\pm 1,2$ anos (CASTILHO; NUCCI, 2015), respectivamente. Estudos internacionais (BANIK, 2011; KAUR; MEHTA; KAUR, 2015) encontraram 12,02 \pm $0,75\left(\mathrm{IC}_{95 \%}: 12,03-12,15\right)$ anos para classes altas e para as classes baixas de 13,70 $\pm 1,31$ anos. O atual estudo encontra concordância com os resultados nacionais e internacionais

Persp. online: biol. \& saúde, Campos dos Goytacazes, 35 (10) 53-62, 2020

https://ojs3.perspectivasonline.com.br 
mostrando que a idade da menarca vem diminuindo, independente da classe econômica (DEMERATH et al., 2013; CARVALHO; FARIAS; GUERRA-JÚNIOR, 2007).

Entretanto, a classe socioeconômica e o tamanho da família mostraram uma associação com a idade da menarca neste estudo em que as meninas de classe alta e famílias pequenas tiveram a menarca mais precocemente do que as suas homólogas de famílias com baixo nível socioeconômico e de famílias maiores. Esse achado é consistente com achados de outros estudos (CARVALHO; FARIAS; GUERRA-JÚNIOR, 2007; LAITINEN; POWER; JARVELIN, 2001).

Apesar de a literatura mostrar que a maturação sexual tende a ocorrer mais precocemente quanto melhor for considerado o nível socioeconômico no qual o indivíduo está inserido, outros estudos mostram resultados diferentes (DENG et al., 2017; YERMACHENKOAND; DVORNYK; NONGENETIC, 2014; MAIO et al., 2014). Em contraste, um estudo do norte da Nigéria não encontrou diferença na média de idade da menarca em relação à classe econômica (TUNAU et al., 2012).

No Brasil, estudos demonstraram tendência à antecipação da menarca mesmo em níveis socioeconômicos menos privilegiados (CASTILHO; NUCCI, 2015). Embora os resultados apresentados no presente estudo abordem que a maturação sexual tende a ocorrer antecipadamente em meninas que pertencem ao nível socioeconômico alto, observa-se que a diferença entre os níveis médio e baixo foi relativamente pequena.

O impacto dos resultados da associação entre a idade da menarca e as classes econômicas bem como o tamanho da família, demonstra que as classes mais favorecidas e de famílias menores, convivem em um ambiente mais farto em alimentos hipercalóricos (ROCHA; ETGES; 2019; THUMÉ; POLL, 2018), estilo de vida mais sedentário afetado pelo avanço exacerbado da tecnologia (aparelhos eletroeletrônicos), colaborando para o aumento da prevalência de excesso de peso (GEMELLI; FARIAS; SOUZA, 2016).

Diante dessa realidade, estudos têm apontado uma associação positiva da idade precoce da menarca com o excesso de peso, observado em meninas de classe econômica mais privilegiada, assim como no presente estudo, em que os aspectos de estilo de vida provavelmente estejam refletindo em tais resultados.

Existem algumas limitações neste estudo. Dentre as limitações do atual estudo, devese ressaltar que o delineamento transversal adotado não permite identificar relações de causalidade entre as variáveis. Não ter avaliado outras variáveis além de fatores extrínsecos, como condições de vida, especialmente em termos de comportamentos psicológicos e hábitos alimentares, bem como por não ter aplicado um questionário adequado para a avaliação do perfil socioeconômico da amostra, e sim avaliado apenas o acesso a bens de consumo.

Apesar das limitações inerentes a todo estudo, alguns pontos fortes merecem ser ressaltados. Embora mais estudos sejam necessários para melhor compreensão entre idade da menarca e os fatores preditores, o presente estudo avança na tentativa de buscar evidências que possam corroborar para o melhor entendimento acerca das implicações da classe socioeconômica e das características antropométricas na época de ocorrência da menarca. Por fim, resultados de pesquisa, em uma amostra de meninas na faixa etária de 10-18 anos pertencentes a um município do norte do Brasil, onde estudos são bastante escassos, são relevantes para contribuir com políticas públicas de promoção da saúde voltadas para a redução da obesidade, adoção de estilo de vida ativo e hábitos alimentares saudáveis. 


\section{CONCLUSÕES}

As meninas de classe social alta têm a IM precoce e são de famílias pequenas. No grupo pós-menarca observou-se estatura, massa corporal e zIMC maiores. Por outro lado, o RIP foi maior no grupo pré-menarca, possuindo linearidade corporal mais adequada, favorecido por uma massa corporal menor, expressando uma estrutura física corporal mais equilibrada, no que se refere à composição corporal, quando comparadas ao grupo pósmenarca.

\section{REFERÊNCIAS}

AHMED, S.M.; WAHEED, M.A.; AHMAD, F.; DANISH, S.H. Factors contributing to early menarche in school girls. Journal Of Pakistan Medical Association , v.66, n.5, p.629-633, 2016.

ASSOCIAÇÃO BRASILEIRA DE EMPRESAS DE PESQUISA. Critério de Classificação Econômica Brasil. São Paulo: ABEP; 2016. 06p. Internet. Disponivel em: $<$ file://C:/Users/UNIR/Downloads/01_cceb_2016_11_04_16_final\%20(3).pdf $>$. Acesso em: 07 fev 2017.

BANIK, S.D. Evaluation of health status of pre-menarcheal and post-menarcheal girls by Rohrer index in Purulia, West Bengal. Journal of Public Health and Epidemiology, v.3, n.1, p.13-16, 2011.

BARROS, B.S.; KUSCHNIR, M.C.M.C; BLOCH, K.V.; SILVA, T.N. ERICA: idade da menarca e sua associação com o estado nutricional. Jornal de Pediatria, 95(1), 106-111, 2019.

CARVALHO, W.R.G.; FARIAS, E.S.; \& GUERRA-JÚNIOR, G. A idade da menarca está diminuindo? Revista Paulista de Pediatria, v.25, n.1, p.76-81, 2007.

CASTILHO, S.D.; \& NUCCI, L.B. Idade da menarca em escolares com e sem excesso de peso. Jornal de Pediatria, v.91, n.1, p.75-80, 2015.

DEMERATH, E.W.; LIU, C.T.; FRANCESCHINI, N. et al. Genome-wide association study of age at menarche in African-American women. Human Molecular Genetics, v.22, n.16, p.3329-3346, 2013.

DENG, X.; L.I.; W.; LUO, Y.; LIU, S. et al. Association between Small Fetuses and Puberty Timing: A Systematic Review and Meta-Analysis. International Journal of Environmental Research, v.14, n.11, p.1377, 2017.

GEMELLI, I.F.B.; FARIAS, E.S.; \& SOUZA, O.F. Age at Menarche and Its Association with Excess Weight and Body Fat Percentage in Girls in the Southwestern Region of the Brazilian Amazon. Journal of Pediatric and Adolescent Gynecology, v.29, p. 482-488, 2016.

KAUR, R.; MEHTA, P.; \& KAUR, G. Growth Status and Menarcheal Age Among Adolescent School Girls of Punjab. International Journal of Recent Scientific Research, v.6, n.7, p.5150-5153, 2015.

LAITINEN, J.; POWER, C.; \& JARVELIN, M.R. Family social class, maternal body mass index, childhood body mass index and age at menarche as predictors of adult obesity. The American Journal of Clinical Nutrition, v.74, n.3, p.287-294, 2001. 
MAGANHIN, C.C.; SIMÕES, R.S.; FUCHS, L.F.P. et al. Melatonin influences on steroidogenic gene expression in the ovary of pinealectomized rats. Fertil Steril, v.102, n.1, p.291-298, 2014.

MAO, S.H.; JIANG, J.; SUN, X. et al. Timing of menarche in Chinese girls with and without adolescent idiopathic scoliosis: current results and review of the literature. European Spine Journal., v.20, n. 2, p. 260-265, 2011.

MARSHALL, W.A.; \& TANNER, J.M. Variation in the pattern of pubertal changes in girls. Archives of Disease in Childhood. v.44, n.235, p.291-303, 1969.

MATCHOCK, R.L.; SUSMAN, E.J.; \& BROWN, F.M. Seasonal rhythms of menarche in the United States: correlates to menarcheal age, birth age, and birth month. Womens Health Issues, v.14, n.6, p.184-192, 2004.

NATIONAL INSTITUTES OF HEALTH. Bioelectrical impedance analysis in body composition measurement: National Institutes of Health Technology Assessment Conference Statement. The American Journal of Clinical Nutrition, v.64, p.524s-532s, 1996.

ROCHA, T. N.; ETGES, B. I. Consumo de alimentos industrializados e estado nutricional de escolares. Revista Perspectivas Online: Biológicas \& Saúde, v. 9, n. 29, p.21-32, 2019.

ROMAN, E.P.; RIBEIRO, R.R.; GUERRA-JÚNIOR, G. et al. Antropometria, maturação sexual e idade da menarca de acordo com o nível socioeconômico de meninas escolares de Cascavel (PR). Revista Assocociação Medica Brasileira, v.55, n.3, p.317-321, 2009.

THUMÉ, C. T., \& POLL, F. A. Qualidade da dieta e fatores de risco para doenças crônicas. Revista Perspectivas Online: Biológicas \& Saúde, v.8, n.26, p.31-41, 2018.

TUNAU, K.A.; ADAMU, A.N.; HASSAN, M.A. et al. Age at menarche among school girls in Sokoto, Northern Nigeria. Annals of African medicine, v.11, p. 103-107, 2012.

WULANDARI, T.; DELIANA, M.; SOFYANI, S. et al. Relationship between age at menarche and exposure to sexual content in audio-visual media and other factors in Islamic junior high school girls. Paediatrica Indonesiana, v.57, n.6, p.323-328, 2017.

YERMACHENKOAND, A.; \& DVORNYK, V.; NONGENETIC. Determinants of Age at Menarche: A Systematic Review. BioMed Research International , v. 2014; ID 371583, p. $14,2014$. 